\title{
Evolución de los pacientes con enfermedad hepática crítica
}

\section{Prognosis of patients with critical hepatic disease}

Juan Higuera Lucas', Raúl De Pablo1, Sergio Sáez Noguero', Sergio Gallego', Lara Rey', Gabriela Narváez Chávez', David Cabestrero Alonso

\begin{abstract}
Introduction: The acute liver failure on chronic (ACLF), is an entity, whose recognition is increasing. The ACLF and CLIF OF indexes have been recently presented with the objective of predicting mortality in this kind of patients. Material and Methods: All patients admitted to the Ramón y Cajal University Hospital diagnosed of acute liver failure on chronic during 2016 and 2017 were collected. We collect the scores: SOFA, CLIF, APACHE II, SAPS II and ACLF score in patients admitted to the ICU by comparing them with each other and define which stages have worse prognosis. Results: A total of 46 patients were collected. The study presents an intra ICU mortality of 31\% (15/46) and a sixmonth mortality of $59.6 \%$ (28/46). Patients classified as death, present ACLF values at admission (49.5 vs $60 p=0.001$ ), and at three days (46.66 vs $59.4 p$ $=0.001$ ) higher than survivors. In the analysis of the ROC curve, the area under the curve in relation to six-month mortality is higher in the ACLF index (0.8) compared to the MELD (0.69) SOFA (0.66) SAPS II (0.69) or APACHE II (0.65). Patients with ACLF indexes above 65 had an intra UCI mortality of 54\%, however, mortality at 6 months is $90 \%$. Patients with ACLF values greater than 65 present mean values of lactic acid, leukocytes, INR or bilirubin higher than those under 65 in a statistically significant manner. Conclusions: The data presented in this study suggest that the ACLF index works as an adequate predictor of intra-ICU mortality and at 6 months.
\end{abstract}

\section{RESUMEN}

Introducción: El fallo hepático agudo sobre crónico es una entidad cuyo reconocimiento va en aumento. Los índices ACLF y CLIF OF, han sido presentados recientemente con el objetivo de predecir la mortalidad en este tipo de enfermos. Material y Métodos: Se recogen todos los pacientes ingresados en

\section{Key words:}

Acute on chronic liver failure, intensive care, scores

\section{Palabras clave:}

Fallo hepático agudo sobre crónico, medicina intensiva, índices de gravedad

Medicina Intensiva, Hospital Universitario Ramón y Cajal.

Fecha de recepción: 09 de julio de 2020

Fecha de aceptación: 17 de julio de 2020

\section{ORCID}

0003-1778-2291

Correspondencia:

Juan Higuera Lucas

jhiguera.151@gmail.com 
una unidad de cuidados intensivos (UCI) de un hospital terciario universitario, diagnosticados de fallo hepático agudo sobre crónico durante 2016 y 2017. Recogemos los índices SOFA, CLIF, APACHE II, SAPS II Y ACLF en pacientes ingresados en UCI comparándolos entre sí. Definimos que estadios presentan peor pronóstico. Resultados: Se analizan un total de 46 pacientes. El estudio presenta una mortalidad intra-UCI del 31\% (15/46) y una mortalidad a los seis meses de 59,6\% (28/46). Los pacientes clasificados como éxitus presentan valores ACLF al ingreso (49,5 vs $60 p=0,001)$, a los tres días $(46,66$ vs 59,4 $p=$ $0,001)$ superiores a los supervivientes. En el análisis de la curva COR, el área bajo la curva en relación a la mortalidad a los seis meses, es superior en el índice $\operatorname{ACLF}(0,8)$ en comparación con el MELD $(0,69) \operatorname{SOFA}(0,66)$ SAPS ॥ $(0,69)$ o APACHE ॥ $(0,65)$. Los pacientes con índices ACLF superiores a 65 presentaban una mortalidad intra-UCI del 54\% sin embargo, la mortalidad a los 6 meses es del $90 \%$. Los pacientes con valores ACLF superiores a 65 presentan a su vez valores medios de láctico, leucocitos, INR o bilirrubina mayores de forma estadísticamente significativa. Conclusiones: Los datos presentados en este estudio sugieren que el índice ACLF funciona como un adecuado predictor de mortalidad intra-UCI y a los 6 meses.

\section{Introducción}

r a cirrosis hepática se define como el desarrollo histológico de nódulos regenerativos rodeados de bandas fibrosas en respuesta a un daño hepático crónico. Esto deriva en hipertensión portal y enfermedad hepática terminal. Los nuevos avances en el conocimiento de la historia natural de la enfermedad, fisiopatología y tratamiento de sus complicaciones, nos ha llevado a un mejor manejo médico de estos pacientes, aumentando su calidad de vida y pronóstico. Aunque la única terapia curativa es el trasplante hepático, el tratamiento farmacológico de la descompensación hepática también puede detener la progresión[1].

El número de pacientes con cirrosis hepática está creciendo en relación al consumo de alcohol, enfermedad hepática no alcohólica o hepatitis víricas. En Estados Unidos, es responsable de aproximadamente 25.000 muertes al año[1],[2]. Inicialmente, estos pacientes, presentan una cirrosis compensada, sin embargo, pueden acabar desarrollando una descompensación aguda deteriorando su enfermedad de base. El sangrado por várices, ascitis, síndrome hepatorrenal, peritonitis bacteriana espontánea y sepsis, son algunas causas de descompensación y pueden ser el motivo de ingreso en unidades de cuidados intensivos (UCIs). El pronóstico y mortalidad en su ingreso en estas unidades ha sido ampliamente descrito[3]-[7]. La mortalidad es elevada, encontrándose entre el $36 \%-86 \%$ y se relaciona de forma significativa con el número de órganos en fallo durante la estancia del paciente en UCI[8]-[10].

El fallo hepático agudo sobre crónico (acute on chronic liver failure -ACLF-) es una entidad cuyo reconocimiento va en aumento. Se caracteriza por una descompensación aguda del paciente con cirrosis previa compensada y estable. Generalmente, presenta fallo de varios órganos y elevada mortalidad[11]. Los criterios diagnósticos y la subsecuente graduación de ACLF, han sido presentados recientemente y están basados en la European Fundation for the study of Chronic Liver Failure (CLIF) organ failure score (CLIFOF) y ACLF en cirrosis (CANONIC)[8]. De acuerdo al estudio CANONIC, ACLF está caracterizado por una descompensación aguda de la cirrosis (ascitis, encefalopatía, hemorragia digestiva, infección bacteriana) asociada con fallo orgánico (hígado, riñón, sistema nervioso central, coagulación, hemodinámico y/o pulmón). El grado de ACLF, se mide conforme al número de órganos en fallo. Existen 3 gradaciones y predice la mortalidad a los 28 días Grado I: 23,3\%; (Grado II: $32 \%$, y Grado III: 75,5\%[12],[13].

Los modelos pronósticos CLIF-C ACLF (chronic liver failure), SOFA (sequential organ failure assessment, MELD (model of end stage liver disease) suelen basarse en las variables medidas en un punto, generalmente al ingreso. ACLF es un proceso dinámico, con reversibilidad en aproximadamente 50\% de los casos o progresión a cronicidad. En los pacientes ingresados en UCl, el SOFA mejora claramente el rendimiento pronóstico del índice tomado al ingre- 
so. Además, es utilizado como parámetro dinámico en seguimiento y evolución del fracaso orgánico. El CLIF-C ACLF puede predecir mejor el pronóstico de los pacientes, mejorar el manejo y minimizar tratamientos fútiles y caros. Sin embargo, se necesita una descripción mejor de este nuevo índice y su asociación al pronóstico[14].

En este estudio, queremos evaluar los índices SOFA, APACHE \|, SAPS \| Y CLIF-C ACLF en pacientes ingresados en UCI comparándolos entre sí. Definir qué estadios presentan peor pronóstico y en qué tipo de descompensación.

Objetivo primario de este estudio: evaluar los índices SOFA, APACHE ॥, SAPS \| Y CLIF-C ACLF en pacientes ingresados en UCl comparándolos entre sí. Como objetivo secundario: Definir qué estadios presentan peor pronóstico y en qué tipo de descompensación.

\section{Material y Métodos}

Se diseña un estudio retrospectivo unicéntrico. Se recogen todos los pacientes ingresados en un Hospital Universitario, terciario y con programa de trasplante hepático, diagnosticados de fallo hepático agudo sobre crónico durante los años 2016 y 2017. Todos los pacientes recogidos recibieron algún apoyo orgánico, incluyendo ventilación mecánica, terapia renal sustitutiva o fármacos vasoactivos.

El estudio cuenta con la aprobación del Comité Ético de Investigación Clínica del Hospital Ramón y Cajal. El tratamiento de los datos ha sido realizado con las medidas de seguridad establecidas en cumplimiento de la Ley Orgánica 15/1999 de Protección de datos de carácter personal.

Las variables analizadas son la edad, días de ingreso, índices SOFA, SAPS II y APACHE II al ingreso; origen de la cirrosis, CHILD previo al ingreso, y si el paciente se encontraba en lista de trasplante. Se analiza la razón del fallo agudo o la causa de su descompensación; la presencia de ascitis o no; los valores de albúmina y creatinina al ingreso y máximo; valor de MELD al ingreso máximo durante toda la estancia intra-UCl; presencia o no de encefalopatía y grado de la misma. Valores de los índices CLIF, CLIF OF y ACLF al ingreso, a los tres días y al alta y la mortalidad predicha por el índice CLIF-C ACLF al mes, tres meses y seis meses. Se recogen el fallo de órgano (ya sea respiratorio, renal, respiratorio, hemodinámico, coagulación o sistema nervioso central); valores de láctico, INR, PCR, leucocitos y bilirrubina al ingreso y máximos durante el mismo; si el paciente precisa, o no, venti- lación mecánica, terapia renal sustitutiva o fármacos vasoactivos para el apoyo de su patología y la mortalidad. Se recoge la mortalidad de los enfermos al mes, tres o seis meses del alta de UCl, y si el paciente acaba siendo trasplantado.

Todos los pacientes recogidos presentaban una hepatopatía crónica en grado de cirrosis compensada hasta el desencadenante que provoca el fallo hepático agudo sobre crónico. Los cálculos de los índices CLIF, CLIF OF y ACLF, se obtienen recogiendo los valores de bilirrubina, creatinina, técnica de reemplazo renal, grado de encefalopatía, INR, presión arterial media, uso o no de fármacos vasoactivos, $\mathrm{PaO}_{2}$, uso de ventilación mecánica, edad y valor de leucocitos en los días mencionados.

Los criterios para el diagnóstico de ACLF fueron hechos utilizando la clasificación CLIF-OF, la cual es una modificación del índice CLIF-C ACLF, SOFA[11],[15]. Los fallos orgánicos son definidos como: Fallo renal, si valor de creatinina mayor o igual a $2 \mathrm{mg} / \mathrm{dl}$ y/o terapia renal sustitutiva. Fallo sistema nervioso central, está definido como encefalopatía grado III/IV conforme a los criterios West Haven. El fallo hepático, se define como valores de bilirrubina mayores o iguales a 12 $\mathrm{mg} / \mathrm{dl}$. La coagulopatía, se define como valores de INR mayores o iguales a 2,5. Fallo hemodinámico, se define como la necesidad de fármacos vasoactivos para mantener tensión arterial media u optimizar gasto cardiaco. El fallo respiratorio, se define como $\mathrm{PaO}_{2} /$ $\mathrm{FiO}_{2}$ menor de 200 .

Así, definiremos también CLIF-C ACLF grado I como la presencia de fallo renal único o cualquier otro órgano combinado con la presencia de fallo renal (Creatinina mayor o igual a 1,5 mg/dl) o encefalopatía hepática grado 1 o 2. CLIF-C ACLF grado 2 y 3, se define por la presencia de dos o al menos tres órganos respectivamente.

Se realiza el análisis estadístico mediante la herramienta IBM ${ }^{\circledR}$ SPSS Statistics 23. La normalidad de las variables ha sido establecida mediante el test de komogorov-Smirnov. Las variables cuantitativas con distribución normal han sido expresadas como media \pm desviación estándar (rango). Estas, han sido comparadas mediante test t-Student o ANOVA. Las distribuciones cuantitativas que no seguían una distribución normal han sido comparadas utilizando test de Wilcoxon y son expresadas como mediana y rango intercuartílico. Las variables cualitativas son mostradas como números y porcentajes. Son comparadas mediante test de McNemar y Chi-cuadrado. El nivel de significación estadística se ha establecido con valores de $\mathrm{P}$ menores de 0,05 . Los resultados son expresados como intervalos de confianza del 95\%. 


\section{Resultados}

Se analizan un total de 46 pacientes. El estudio presenta una mortalidad intra-UCI del 31\% (15/46) y una mortalidad a los seis meses de 59,6\% (28/46). Las características de los pacientes se presentan en la Tabla 1. Sólo un paciente fue trasplantado en nuestra muestra. De los 46 pacientes incluidos, los valores de creatinina máximo $(\mathrm{mg} / \mathrm{dl})$, bilirrubina máximo (mg/ dl), máximo valor de ácido láctico, fueron mayores en pacientes que mueren. Sin embargo, el valor leucocitario o el valor de INR al ingreso, no se asocian a la mortalidad de forma estadísticamente significativa.
En el análisis comparativo de los pacientes que mueren durante su estadía en $\mathrm{UCl}$, el valor medio de leucocitos en los pacientes con fallo agudo sobre crónico, también se asocia con la mortalidad ( $p=0,008$ ). Además, se asocia el uso de ventilación mecánica ( $p=$ $0,025)$, terapia de reemplazo renal $(p=0,02)$ y uso de fármacos vasoactivos $(p=0,022)$.

En nuestro estudio, 12 pacientes presentaban una hepatopatía crónica en grado cirrosis debido a hepatitis vírica $(B, C) ; 23$ pacientes presentaban causa enólica; 4 presentaban otros tipos (cirrosis biliar primaria, hepatopatía no alcohólica o autoinmune); 7 pacientes presentan cirrosis de causa alcohólica y

\begin{tabular}{|c|c|c|c|}
\hline Parámetro & Vivo & Éxitus & $\mathbf{P}$ \\
\hline$n$ & 15 & 31 & \\
\hline Edad & 51 & 58,1 & $p=0,007$ \\
\hline Días de estancia & 12,8 & 8,85 & $p=0,35$ \\
\hline SOFA & 9,33 & 11,75 & $p=0,11$ \\
\hline APACHE ॥ & 18 & 22 & $p=0,136$ \\
\hline SAPS ॥ & 42,6 & 53,75 & $p=0,052$ \\
\hline MELD ingreso & 18,11 & 24,6 & $p=0,03$ \\
\hline MELD máximo & 21,4 & 29,1 & $p=0,016$ \\
\hline Creatinina ingreso (mg/dl) & 1,35 & 1,95 & $p=0,182$ \\
\hline Creatinina máxima (mg/dl) & 1,5 & 2,4 & $p=0,074$ \\
\hline CLIF grado ingreso & 2,25 & 2,48 & $p=0,4$ \\
\hline CLIF OF ingreso & 10,5 & 12,1 & $p=0,068$ \\
\hline ACLF score ingreso & 49,5 & 60 & $p=0,001$ \\
\hline CLIF grado 3 días & 2 & 2,72 & $p=0,009$ \\
\hline CLIF OF 3 días & 8,77 & 12,08 & $p=0,001$ \\
\hline ACLF 3 días & 46,66 & 59,4 & $p<0,0001$ \\
\hline CLIF grado alta & 1,25 & 2,56 & $p=0,005$ \\
\hline CLIF OF alta & 7,44 & 12,1 & $p<0,0001$ \\
\hline ACLF alta & 42,3 & 62,1 & $p<0,0001$ \\
\hline Láctico ingreso & 2,3 & 4,6 & $p=0,12$ \\
\hline Láctico máximo & 2,4 & 6 & $p=0,03$ \\
\hline Bilirrubina ingreso & 5,8 & 8,8 & $p=0,23$ \\
\hline INR ingreso & 1,66 & 2,81 & $p=0,27$ \\
\hline Leucocitos ingreso & 8.160 & 11.894 & $p=0,039$ \\
\hline Ventilación mecánica & $72,22 \%$ & $85,7 \%$ & $p=0,227$ \\
\hline Terapia renal sustitutiva & $11,1 \%$ & $53,6 \%$ & $p=0,004$ \\
\hline Fármacos vasoactivos & $55,5 \%$ & $78,6 \%$ & $p=0,09$ \\
\hline
\end{tabular}




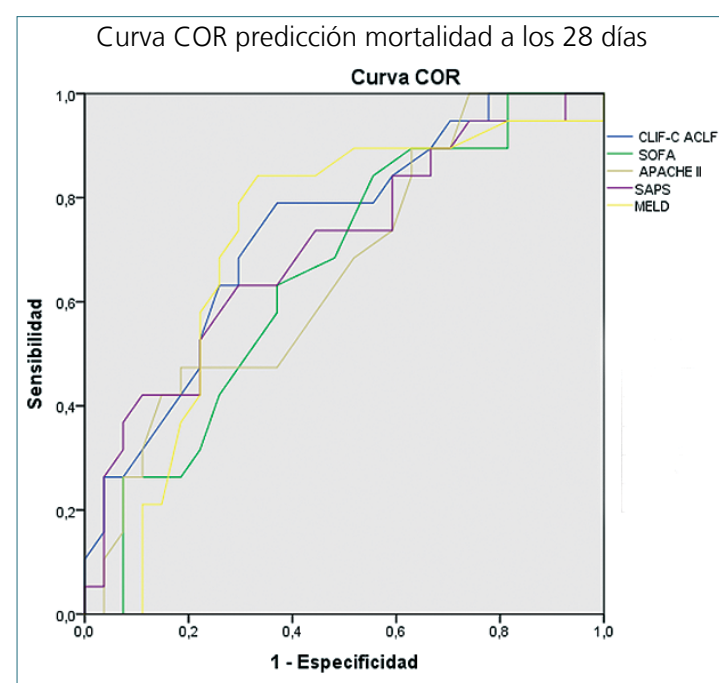

Curva COR mortalidad a los 3 meses

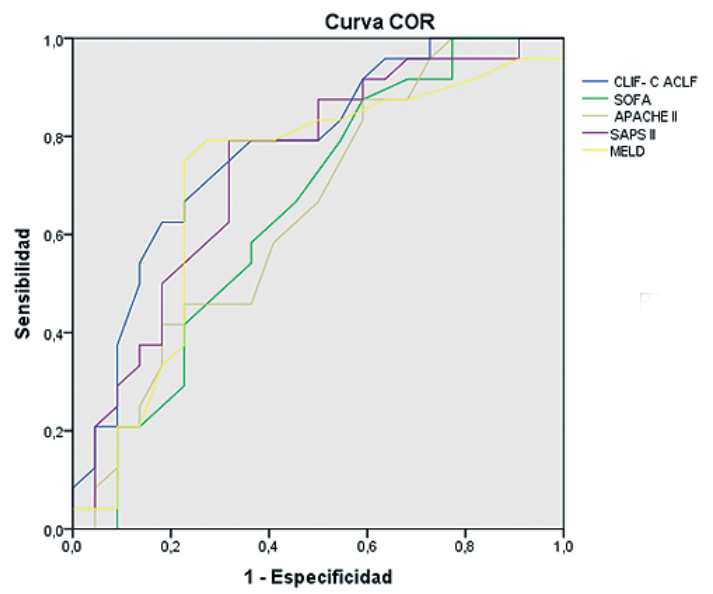

Curva COR predicción mortalidad a los 6 meses

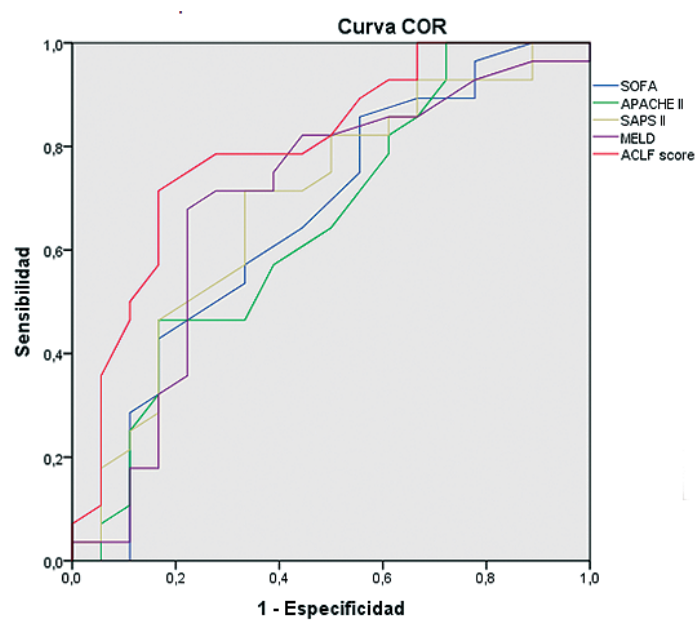

Tabla 2. Curva COR de los diferentes índices pronósticos en fallo hepático agudo sobre crónico. Se representa el pronóstico a los 28 días, 3 meses y 6 meses. El índice ACLF presenta el mayor valor predictivo para la mortalidad a los seis meses en este tipo de pacientes

$\begin{array}{lc}\text { Parámetro } & \text { Área bajo la curva } \\ \text { ACLF } & 0,736 \\ \text { SOFA } & 0,654 \\ \text { APACHE II } & 0,66 \\ \text { SAPS II } & 0,7 \\ \text { MELD } & 0,713\end{array}$

\section{Parámetro}

ACLF

SOFA

APACHE ॥

SAPS ॥

MELD

\section{Área bajo la curva}
0,776
0,65
0,65
0,73

0,7

\section{Parámetro}

ACLF

SOFA

APACHE ॥

SAPS ॥

MELD

\section{Área bajo la curva}

0,8

0,659

0,648

0,69

0,69 
vírica asociadas.

Los pacientes presentaron como causa de descompensación: sangrado en 22 ocasiones, infecciosa en 15 ocasiones, neurológica en 5 ocasiones, insuficiencia respiratoria 2, hepatitis aguda alcohólica 1 y otras causas en 2.

La curva COR en relación a predicción de mortalidad a los seis meses, presenta un área bajo la curva de 0,8 en el CLIF-C ACLF score, 0,69 en SAPS II y MELD, 0,658 en SOFA y 0,648 en APACHE II (Tabla 2). El área bajo la curva en las curvas COR, también es mayor con el índice CLIF-C ACLF en cuanto a la mortalidad intra-UCI con un área bajo la curva de 0,763. En la Tabla 2 se representan, además, los valores de la curva COR en relación a predicción de mortalidad a los 28 días y tres meses.

Se divide a los pacientes según el índice CLIF-C ACLF en función del valor del índice. Se establecen 5 grupos: Menos de 55, mayor o igual a 55, mayor o igual a 60 , mayor igual a 65 o mayor o igual a 70 . Los resultados se exponen en la Tabla 3.

La supervivencia intra-UCl es del $77 \%$ en el grupo de pacientes con índices CLIF-C ACLF menores de 65, mientras que, en aquellos con números mayores, la supervivencia baja a $45 \%(P=0,056)$. En la Tabla 4 se muestran las características de los enfermos con valores CLIF-C ACLF mayores y menores de 65.

\section{Discusión}

Los datos presentados en este estudio, sugieren que el índice CLIF-C ACLF, funciona como un predictor adecuado de mortalidad intra $\mathrm{UCI}$ y a los 6 meses. En nuestra muestra es mejor predictor de mortalidad que los otros índices de gravedad usados en los enfermos críticos como el SOFA, APACHE II, SAPS II o los específicos como el MELD, para los enfermos con fallo hepático agudo sobre crónico. Cuando su valor supera el grado 3 (65) la mortalidad a los 6 meses objetivada es del $90 \%$. Engelmann et al.[14] proponen resultados similares llegando hasta el $94 \%$ de mortalidad a los 28 días con valores superiores o iguales a 65.

Los pacientes con fallo hepático agudo sobre crónico con índices CLIF-C ACLF superiores a 55, presentan una mortalidad intra-UCl en nuestra muestra de $48 \%$ y a los 6 meses del $84 \%$. Valores superiores al 65 pronostican una mortalidad del $90 \%$ aunque intra-UCI la mortalidad sea del $54 \%$. Estos datos, sin poder ser generalizables dado que el estudio es unicéntrico, nos demuestran que pese al máximo tratamiento intensivo que nuestros pacientes reciben en las unidades de cuidados intensivos, el número de pacientes que fallece tras el alta, en los primeros seis meses es muy elevado. En muchos casos el tratamiento intensivo pudiera parecer fútil. Este índice, requiere reevaluación por medio de otros estudios multicéntricos dado que puede cambiar la valoración del pronóstico y el curso evolutivo de estos enfermos.

Esta diferencia de mortalidad intra-UCl y a los seis meses, puede reflejar la limitada reserva y capacidad de regeneración que tiene el paciente cirrótico tras una reagudización. Mientras sus órganos se encuentran bajo apoyo hemodinámico, respiratorio o renal, el paciente sobrevive, pero conforme a los datos mostrados, tras su retirada, la capacidad de regeneración es escasa.

Una de las opciones curativas con las que cuentan los pacientes es con el trasplante hepático. Nuestro hospital es centro de referencia de trasplante. En algunas series, se describe una supervivencia al año mayor del $80 \%$ en ausencia de fallo hepático agudo sobre crónico[16]. Sin embargo, en los pacientes trasplantados tras reagudización de su fallo crónico, la supervivencia puede bajar a 43\%[17]. En nuestra muestra, sólo un paciente con fallo hepático agudo sobre crónico fue trasplantado, con un índice ACLF de 59 y resultó éxitus a los 6 meses. Estos resultados plantean la pregunta si el trasplante hepático en estas situaciones pudiera ser beneficioso. Nuevamente, se necesitarían más datos sobre trasplantes realizados a

\begin{tabular}{ccc}
\multicolumn{3}{c}{ Tabla 3. Mortalidad en función valor índice CLIF-C ACLF } \\
\hline CLIF-C ACLF & Mortalidad intra-UCI & Mortalidad 6 meses \\
$<55$ & $9,5 \%(2 / 21)$ & $33,3 \%(7 / 21)$ \\
$\geq 55$ & $48 \%(12 / 25)$ & $84 \%(21 / 25)$ \\
$\geq 60$ & $47,4 \%(9 / 19)$ & $84 \%(16 / 19)$ \\
$\geq 65$ & $54 \%(6 / 11)$ & $90 \%(10 / 11)$ \\
$\geq 70$ & $66,66 \%(4 / 6)$ & $83,33 \%(5 / 6)$ \\
\hline
\end{tabular}




\begin{tabular}{|c|c|c|c|}
\hline Parámetro & CLIF-C ACLF $<65$ & CLIF-C ACLF > 65 & $\mathbf{P}$ \\
\hline$n$ & 35 & 11 & \\
\hline Edad & 53,9 & 60 & $P=0,047$ \\
\hline SOFA & 9,7 & 14,3 & $P=0,006$ \\
\hline APACHE ॥ & 18,8 & 25,8 & $P=0,021$ \\
\hline SAPS ॥ & 45,5 & 61 & $P=0,012$ \\
\hline Días de ingreso & 11,5 & 7 & $P=0,36$ \\
\hline MELD ingreso & 19,5 & 30 & $P=0,001$ \\
\hline MELD Máximo & 22,8 & 36,4 & $P<0,0001$ \\
\hline Creatinina ingreso & 1,6 & 2 & $P=0,5$ \\
\hline Creatinina máxima & 1,94 & 2,4 & $P=0,4$ \\
\hline CLIF grado & 2,17 & 2,91 & $P=0,004$ \\
\hline CLIF OF & 10,4 & 14,8 & $P<0,0001$ \\
\hline ACLF ingreso & 51,5 & 69,8 & $P<0.0001$ \\
\hline Ácido láctico ingreso & 2,5 & 8,7 & $P<0,0001$ \\
\hline Láctico máximo & 3,5 & 8,8 & $P=0,006$ \\
\hline Bilirrubina ingreso & 5,2 & 15,4 & $P<0,0001$ \\
\hline Bilirrubina máxima & 9,78 & 17,9 & $P=0,026$ \\
\hline INR ingreso & 1,74 & 4,34 & $P=0,028$ \\
\hline Leucocitos ingreso & 9.352 & 13.872 & $P=0,028$ \\
\hline Leucocitos máximo & 15.827 & 19.000 & $P=0,218$ \\
\hline Ventilación mecánica & $74,2 \%$ & $100 \%$ & $P=0,064$ \\
\hline Terapia renal & $31,4 \%$ & $54,5 \%$ & $P=0,152$ \\
\hline F. Vasoactivos & $62,8 \%$ & $90 \%$ & $P=0,077$ \\
\hline Mortalidad intra-UCI & $22,8 \%$ & $54,5 \%$ & $P=0,056$ \\
\hline Mortalidad 6 meses & $51,4 \%$ & $90 \%$ & $P=0,019$ \\
\hline
\end{tabular}

un número mayor de enfermos con un fallo hepático agudo sobre crónico con índices elevados de ACLF.

En nuestra muestra, dentro de las medidas de soporte que más se asocia a la mortalidad es la terapia renal sustitutiva (TRS). El paciente que requiere TRS presenta mayor mortalidad. Dentro de los pacientes fallecidos el 53,6\% fue dializado, mientras que tan solo lo requieren el $11,1 \%$ de los supervivientes. Otras terapias como el apoyo hepático extracorpóreo (molecular adsorbent recirculation system MARS), tampoco ha demostrado claro beneficio[18].

Es importante destacar que los pacientes con índices CLIF-C ACLF superiores a 65, presentan valores más altos de láctico y leucocitos, de forma estadísticamente significativa. Esto, puede reflejar una respuesta inflamatoria mayor en los pacientes más graves. Estos resultados también son descritos por Enegelmann et al., con valores ACLF superiores a 70[14] y por Claria et al.[19], sugiriendo que estrategias para control y disminución de la inflamación podrían mejorar el pronóstico de estos pacientes. En este apartado, hay que tener en cuenta que el hígado es el encargado de realizar hasta el $60 \%$ del metabolismo del lactato. En el fallo hepático, los valores elevados de lactato arterial pueden deberse no sólo a un aumento de producción, sino también a una disminución de su metabolismo por enfermedad hepática[20].

El estudio mostrado presenta importantes limitaciones que deben tenerse en cuenta: en primer lugar, es un análisis unicéntrico y retrospectivo. Sin embargo, los resultados mostrados pueden ayudar a continuar con la valoración de herramientas que permitan 
describir mejor el pronóstico, nos ayuden a tomar decisiones apropiadas y adecuadas o a evitar tratamientos fútiles.

\section{Conclusiones}

Los datos presentados en este estudio sugieren que el índice CLIF-C ACLF, funciona como un adecuado predictor de mortalidad intra-UCl y a los 6 meses. En nuestra muestra, es mejor predictor de mortalidad que otros índices como el SOFA, APACHE II, SAPS II o MELD, en el paciente con fallo hepático agudo sobre crónico.

Valores superiores al 65 pronostican una mortalidad del $90 \%$ a los 6 meses. Este índice pudiera ayudar a discriminar, en ausencia de trasplante, si la continuidad de tratamiento invasivo es útil. Son necesarios más estudios para afirmar esto.

\section{Referencias}

1. chuppan $\mathrm{D}$, Afdhal $\mathrm{NH}$. Liver cirrhosis. Lancet. 2008 Mar;371(9615):838-51. https://doi.org/10.1016/ S0140-6736(08)60383-9 PMID:18328931

2. Levesque E, Saliba F, Ichaï $P$, Samuel D. Outcome of patients with cirrhosis requiring mechanical ventilation in ICU. J Hepatol. 2014 Mar;60(3):570-8. https://doi. org/10.1016/j.jhep.2013.11.012 PMID:24280294

3. Shellman RG, Fulkerson WJ, DeLong E, Piantadosi CA. Prognosis of patients with cirrhosis and chronic liver disease admitted to the medical intensive care unit. Crit Care Med. 1988 Jul;16(7):671-8. https:// doi.org/10.1097/00003246198807000-00005 PMID:3371043

4. Singh N, Gayowski T, Wagener MM, Marino IR. Outcome of patients with cirrhosis requiring intensive care unit support: prospective assessment of predictors of mortality. J Gastroenterol. 1998 Feb;33(1):73-9. https://doi. org/10.1007/s005350050047 PMID:9497225

5. Aggarwal A, Ong JP, Younossi ZM, Nelson DR, Hoffman-Hogg L, Arroliga AC. Predictors of mortality and resource utilization in cirrhotic patients admitted to the medical ICU. Chest. 2001 May;119(5):1489-97. https://doi. org/10.1378/chest.119.5.1489 PMID:11348958

6. Chen YC, Tsai MH, Ho YP, Hsu CW, Lin HH, Fang JT, et al. Comparison of the severity of illness scoring systems for critically ill cirrhotic patients with renal failure. Clin Nephrol. 2004 Feb;61(2):111-8. https:// doi.org/10.5414/CNP61111 PMID:14989630

7. Shawcross DL, Austin MJ, Abeles $R D, M c P h a i l$ MJ, Yeoman AD, Taylor NJ, et al. The impact of organ dysfunction in cirrhosis: survival at a cost? J Hepatol. 2012 May;56(5):1054-62. https://doi. org/10.1016/j.jhep.2011.12.014 PMID:22245890

8. Moreau R, Jalan R, Gines $P$, Pavesi M, Angeli P, Cordoba J, et al.; CANONIC Study Investigators of the EASL-CLIF Consortium. Acute-on-chronic liver failure is a distinct syndrome that develops in patients with acute decompensation of cirrhosis. Gastroenterology. 2013 Jun;144(7):142637. https://doi.org/10.1053/j. gastro.2013.02.042 PMID:23474284

9. Saliba F, Ichai $P$, Levesque $E$, Samuel D. Cirrhotic patients in the ICU: prognostic markers and outcome. Curr Opin Crit Care. 2013 Apr;19(2):154-60. https://doi.org/10.1097/ MCC.0b013e32835foc17
PMID:23426137

10. Galbois A, Das V, Carbonell N, Guidet B. Prognostic scores for cirrhotic patients admitted to an intensive care unit: which consequences for liver transplantation? Clin Res Hepatol Gastroenterol. 2013 Nov;37(5):455-66. https://doi.org/10.1016/j.clinre.2013.05.001 PMID:23773487

11. Jalan $R$, Gines $P$, Olson JC, Mookerjee RP, Moreau R, Garcia-Tsao G, et al. Acute-on chronic liver failure. J Hepatol. 2012 Dec;57(6):1336-48. https://doi. org/10.1016/j.jhep.2012.06.026 PMID:22750750

12. Gustot $T$, Fernández J, García E, Morando F, Caraceni P, Alessandria $C$, et al.; CANONIC Study Investigators of the EASL-CLIF Consortium. Clinical Course of acute-on-chronic liver failure syndrome and effects on prognosis. Hepatology. 2015 Jul;62(1):24352. https://doi.org/10.1002/ hep.27849 PMID:25877702

13. Jalan $R$, Saliba F, Pavesi $M$, Amoros $A$, Moreau R, Ginès $P$, et al.; CANONIC study investigators of the EASL-CLIF Consortium. Development and validation of a prognostic score to predict mortality in patients with acute-on-chronic liver failure. J Hepatol. 2014 Nov;61(5):1038-47. https://doi. org/10.1016/j.jhep.2014.06.012 PMID:24950482

14. Engelmann C, Thomsen $\mathrm{KL}$, 
Zakeri N, Sheikh M, Agarwal $B$, Jalan $R$, et al. Validation of CLIF-C ACLF score to define a threshold for futility of intensive care support for patients with acute-on-chronic liver failure. Crit Care. 2018 Oct;22(1):254. https://doi.org/10.1186/s13054018-2156-0 PMID:30305132

15. Sarin SK, Kumar A, Almeida JA, Chawla YK, Fan ST, Garg $\mathrm{H}$, et al. Acute-on-chronic liver failure: consensus recommendations of the Asian Pacific Association for the study of the liver (APASL). Hepatol Int. 2009 Mar;3(1):269-82. https://doi. org/10.1007/s12072-008-9106-x PMID:19669378

16. Artru F, Louvet A, Ruiz I, Levesque E, Labreuche J, Ursic-Bedoya J, et al. Liver transplantation in the most severely ill cirr- hotic patients: A multicenter study in acute-on-chronic liver failure grade 3. J Hepatol. 2017 Oct;67(4):708-15. https://doi. org/10.1016/j.jhep.2017.06.009 PMID:28645736

17. Levesque $E$, Winter $A$, Noorah Z, Daurès JP, Landais P, Feray $C$, et al. Impact of acute-onchronic liver failure on 90-day mortality following a first liver transplantation. Liver Int. 2017 May;37(5):684-93. https:// doi.org/10.1111/liv.13355 PMID:28052486

18. Hessel FP, Bramlage P, Wasem J, Mitzner SR. Cost-effectiveness of the artificial liver support system MARS in patients with acute-on-chronic liver failure. Eur J Gastroenterol Hepatol. 2010 Feb;22(2):213-20. https://doi.org/10.1097/
MEG.0b013e3283314e48

PMID:19773666

19. Clària J, Stauber RE, Coenraad MJ, Moreau R, Jalan R, Pavesi $M$, et al.; CANONIC Study Investigators of the EASL-CLIF Consortium and the European Foundation for the Study of Chronic Liver Failure (EF-CLIF). Systemic inflammation in decompensated cirrhosis: characterization and role in acute-on-chronic liver failure. Hepatology. 2016 Oct;64(4):1249-64. https:// doi.org/10.1002/hep. 28740 PMID:27483394

20. Hernández G, Bellomo R, Bakker J. The ten pitfalls of lactate clearance in sepsis. Intensive Care Med. 2019 Jan;45(1):82-5. https://doi.org/10.1007/s00134018-5213-x PMID:29754310 